\title{
SOME RESULTS FOR ANALYZING TIME-DEPENDENT FREQUENCIES OF VIBRATION SIGNALS
}

\author{
Tran DuONG TRI \\ Institute of Mechanics, NCST of Vietnam
}

\begin{abstract}
This paper provides some results for analyzing relations between frequencies and time of vibration signals. These results have been obtained by studying the properties of wavelet transform, the spectral analysis, the Short-time Fourier transform and by using the toolboxes in the software parked MATLAB. We have created the corresponding PC programs in order to realize algorithms and for the illustration of results by exploring examples.
\end{abstract}

\section{Introduction}

For analyzing vibrations signal of machine details, constructions... one of the main interested problems is that how to specify the time-frequency relations of that signal. We known, that the Fourier transform allows to establish relations between amplitudes and frequencies only.

In last years, the research results of wavelet transform and its applications in the differential domains: functional analysis, signal processing...are interested by several authors [1], [2], [3], [4]. However, the applications of the wavelet transform for analysing vibration signals have been little studied. In this paper, we present some initial results in applications of the wavelet transform for analyzing vibration signals. The obtained results had been built in the graphical forms, which are in PC programs. We have written these PC programs in MATLAB language. The main problem is given by the illustrations of relations between frequencies and time of vibration signals

\section{A Property of the Wavelet Transform}

\subsection{Definitions}

Definition 1. A function $\psi$ is defined in the interval $R=(-\infty,+\infty)$ that is the wavelet if the following conditions are satisfied

$$
\begin{gathered}
\psi \in L^{2}(R) \text { and } 0<C_{\psi}:=2 \pi \int_{R} \frac{|\hat{\psi}(\omega)|^{2}}{|\omega|} d \omega<\infty \\
\hat{\psi}(\omega)=\lim _{n \rightarrow \infty} \frac{1}{\sqrt{2 \pi}} \int_{-0}^{n} \psi(t) e^{-i \omega t} d t
\end{gathered}
$$


is the Fourier transform of the function $\psi(t)$, and $L^{2}(R)$ is the space of the square integrable functions which are defined on $R$.

Definition 2. Let $\psi$ be a wavelet, the signal $f$ is a function of time $t$, and $f \in L^{2}(R)$. The Wavelet transform of the function $f$ and wavelet $\psi$ - is symbolized as $L_{\psi} f$ - is defined by the following expression

$$
L_{\psi} f(a, b)=\frac{1}{\sqrt{C_{\psi}}}|a|^{-1 / 2} \int_{R} f(t) \psi\left(\frac{t-b}{a}\right) d t, \quad \forall a \in R \backslash\{0\}, \forall b \in R .
$$

That means, when the wavelet $\psi$ and the signal $f \in L^{2}(R)$ are given, from (2.3) $L_{\psi} f$ defines an image of operator

$$
L_{\psi}: L^{2}(R) \ni f \rightarrow L_{\psi} f \in L^{2}((R \backslash\{0\}) \times R) .
$$

Existence of the inverse operator, $\left(L_{\psi}\right)^{-1}$, as well as its applications is usually a problem that is interested in research with respect to the definition of an operator.

\subsection{The Inverse Wavelet Transform}

In the applications fields of signal processing, the inverse Wavelet transform always is used to reconstruct the initial signal from the results, which had been obtained by the Wavelet transform. In [1], [2], the proof of the inverse Wavelet transform formula is based on fundamentals of group theory and the functional analysis. In this section, we propose a proof "directly".

Theorem 1 (Inverse Wavelet transform formula). If $\psi$ is a wavlet, $f \in L^{2}(R)$ and

$$
L_{\psi} f(a, b)=\frac{1}{\sqrt{C_{\psi}}}|a|^{-1 / 2} \int_{R} f(t) \psi\left(\frac{t-b}{a}\right) d t, \quad \forall a \in R \backslash\{0\}, \forall b \in R
$$

then

$$
f(t)=C_{\psi}^{-1 / 2} \iint_{R} L_{\psi} f(a, b) \frac{1}{\sqrt{|a|}} \psi\left(\frac{t-b}{a}\right) \frac{1}{a^{2}} d a d b
$$

We have the following supported results, which are obtained from

1. Fourier transform:

1.1. If $\varphi(t) \leftrightarrow$ (Its Fourier transform is) $\hat{\varphi}(\omega)$ then

$$
\varphi(t-a) \leftrightarrow e^{-i \omega a} \hat{\varphi}(\omega)
$$

1.2. If $\varphi_{1}(t) \leftrightarrow \hat{\varphi}_{1}(\omega), \varphi_{2}(t) \leftrightarrow \hat{\varphi}_{2}(\omega)$ and $\bar{\varphi}(t)$ is the complex conjugate of $\varphi(t)$ then

$$
\int_{R} \varphi_{1}(t) \bar{\varphi}_{2}(t) d t=\int_{R} \hat{\varphi}_{1}(\omega) \overline{\hat{\varphi}}_{2}(\omega) d \omega
$$


2. definition of the delta-function $\delta(t)$ :

2.1. With a continuous function $x(t)$ at all points of $R, t_{0} \in R$, we have:

$$
\int_{R} x(t) \delta\left(t-t_{0}\right) d t=\int_{R} x(t) \delta\left(t_{0}-t\right) d t=x\left(t_{0}\right)
$$

2.2. The Inverse Fourier transform of delta function $\delta(t)$ has the form:

$$
\delta(t)=\frac{1}{2 \pi} \int_{R} e^{i \omega t} d \omega
$$

Proof. We have

$$
\begin{aligned}
I & :=C_{\psi}^{-1 / 2} \iint_{R} L_{\psi} f(a, b) \frac{1}{\sqrt{|a|}} \psi\left(\frac{x-b}{a}\right) \frac{1}{a^{2}} d a d b \\
& =C_{\psi}^{-1} \iint_{R}|a|^{-3} \psi\left(\frac{t-b}{a}\right) \psi\left(\frac{x-b}{a}\right) d t d a d b \\
& =C_{\psi}^{-1} \int_{R} f(z)\left(\iint_{R}|a|^{-3} \psi\left(\frac{z-b}{a}\right) \psi\left(\frac{x-b}{a}\right) d a d b\right) d z=C_{\psi}^{-1} \int_{R} f(z) A d z
\end{aligned}
$$

where

$$
A:=\iint_{R}|a|^{-3} \psi\left(\frac{z-b}{a}\right) \psi\left(\frac{x-b}{a}\right) d a d b=\int_{R}|a|^{-3}\left(\int_{R}\left(\frac{z-b}{a}\right) \psi\left(\frac{x-b}{a}\right) d b\right) d a
$$

Putting $u=\frac{x-b}{a} \rightarrow b=x-a u, d b=-a d u$. Since $\psi \in L^{2}(R), \psi=\bar{\psi}$, from (2.6) and (2.5), we have

$$
\begin{aligned}
A & =\int_{R}|a|^{-2}\left(\int_{R} \psi(u) \bar{\psi}\left(u-\frac{x-z}{a}\right) d u\right) d a=\int_{R}|a|^{-2}\left(\int_{R}|\hat{\psi}(\omega)|^{2} e^{i \omega\left(\frac{x-z}{a}\right)} d \omega\right) d a \\
& =\int_{R}|\hat{\psi}(\omega)|^{2}\left(\int_{R}|a|^{-2} e^{i \omega\left(\frac{x-z}{a}\right)} d a\right) d \omega=\int_{R}|\hat{\psi}(\omega)|^{2} B d \omega
\end{aligned}
$$

where

$$
B=\int_{R}|a|^{-2} e^{i \omega\left(\frac{x-z}{a}\right)} d a=\int_{-\infty}^{0}|a|^{-2} e^{i \omega\left(\frac{x-z}{a}\right)} d a+\int_{0}^{\infty}|a|^{-2} e^{i \omega\left(\frac{x-z}{a}\right)} d a .
$$

Let us the substitution $\omega / a=u \rightarrow d a=-\left(\omega d u / u^{2}\right)$ then 


$$
\begin{aligned}
B & =\int_{0}^{-\infty} \frac{e^{i u(x-z)}}{(\omega / u)^{2}}\left(-\frac{\omega d u}{u^{2}}\right)+\int_{\infty}^{0} \frac{e^{i v z(x-z)}}{(\omega / u)^{2}}\left(-\frac{\omega d u}{u^{2}}\right) \\
& =\int_{-\infty}^{0} \frac{e^{i u(x-z)}}{|\omega|} d u+\int_{0}^{\infty} \frac{e^{i u(x-z)}}{|\omega|} d u=\frac{1}{|\omega|} \int_{-\infty}^{\infty} e^{i u(x-z)} d u \stackrel{(2.8)}{=} \frac{1}{|\omega|} 2 \pi \delta(x-z) .
\end{aligned}
$$

Therefore

$$
A=\left(\int_{R} \frac{|\hat{\psi}(\omega)|^{2}}{|\omega|} d \omega\right) 2 \pi \delta(x-z)=C_{\psi} \delta(x-z)
$$

Then

$$
I=C_{\psi}^{-1} \int_{R} f(z) C_{\psi} \delta(x-z) d z=\int_{R} f(z) \delta(x-z) d z \stackrel{(2.7)}{=} f(x)
$$

the formula (2.4) has been proved.

From this proof, we shall obtain the following property of the wavelet $\psi$.

If $\psi$ is a wavelet then

$$
\iint_{R} \psi(u) \psi\left(u-\frac{x-z}{a}\right) \frac{d u d a}{a^{2}}=C_{\psi} \delta(x-z) .
$$

\section{Applications of Wavelet transform}

The Wavelet transform has many applications in the different fields [1], [2], [3], [4]. Here we try to present some applications of Wavelet transform to analyze signals that is used to describe vibration phenomenon. Analyzing the signals we are interested in problems of illustrating changes of frequencies according to time in the signal. If Fourier transform is used for analyzing the signals, then in the frequency domain, time information will be lost. Looking at a Fourier transform of a signal, it is impossible to tell when and at what frequencies a signal occurs. In this section, we illustrate changes of frequencies according to time in vibration signal processing. At the same time, the Short Time Fourier Transform (STFT) has been used also to present the similar results here. Both above mentioned methods have been realized by using our PC programs and by graphical analysis. The source codes of the PC programs have been written in MATLAB language, which can run in the environment of MATLAB.

In this paper, wavelet analysis for a signal $s(t)$ means that we must first expand the signal $s(t)$ into the sum of wavelet components - the same harmonics components 
in Fourier expansion of any signal - and then the vibration information of the given signal will be studied through analysis of these components.

\subsection{Analyzing time-dependent frequencies of a signal by Wavelet trans- form}

3.1.1. Remarks. For analyzing time-dependent frequencies, we have built the following analytical method:

The first, a signal is decomposed by Wavelet transform into the sum of component signals. An effective tool supported in the analytical process is the Wavelet Toolbox (Wavelet 1-D) in MATLAB software. Using the Wavelet 1-D, with corresponding selected-parameters, we obtain the table of graphics. These graphics represent the results of signal decomposing into the sum of component signals.

The second, the signal is analyzed to find the relations between frequencies in the signal and time. We perform these analyses from component signals. However, from wavelet 1-D we do not explain which frequencies the signal contains. Even so, using the analysis, wavelet 1-D still makes the presence of "extra" frequencies. The "extra" frequencies obtained in analyzed process are not the frequencies of the given signal. Therefore, the spectral analysis method is used to select frequencies in the given signal.

Using STFT method, we also obtained the similar analyzed results. Comparisons between two methods are not discussed in this paper (see. in [1], [2], [3], [4]).

So, an analyzed process for relations of time-frequencies of a signal includes:

Step 1. Spectra analyzing a given signal (the Fast Fourier Transform is used) will give all frequency components that are embedded in the signal.

Step 2. Wavelet analyzing a given signal will indicate relations between the obtained frequencies and time.

We will present the steps of analysis in the following combination.

\subsubsection{Combination Analysis}

In the following examples, we shall apply the spectral analysis method for signals that are the discrete-time functions. The frequencies found do not depend on the particular analytical expression of given signal.

Example 3.1.1. The signal $s(t)$ is given by the following expressions:

$$
s(t)=\sin (2 \pi 50 t)+\sin (2 \pi 120 t) .
$$

Thus, the vibration signal $s(t)$ is the sum of two sinusoid harmonic oscillations which $f_{1}=50 \mathrm{~Hz}$ and $f_{2}=120 \mathrm{~Hz}$ are the respective frequencies. We shall choose the sampling frequency $f_{C}=1000 \mathrm{~Hz}$ and time $(s) t \in[0,1.0]$.

PC program ARFT321.m give us the results in figures 1, 2, 3 and 4.

The amplitude spectra graphic of signal $s(t)$ is given in figure 2 .

In figure 2 we show the amplitude spectra $|A(f)|$ of signal $s(t)$. This spectra has two major values, $f_{1}=50 \mathrm{~Hz}, f_{2}=120 \mathrm{~Hz}$ and $A\left(f_{1}\right)=0.99635, A(f 2)=0.97500$. 
So the given signal $s(t)$ has the frequency components $f_{1}=50 \mathrm{~Hz}$ and $f_{2}=120 \mathrm{~Hz}$. The wavelet analyzed graphics are presented in figure 3 .

From figure 3, we have the following remarks:

1. The oscillation signal $s(t)$ is decomposed into the sum of the component oscillations $A_{5}, D_{1}, D_{2}, D_{3}, D_{4}$ and $D_{5}$ that is $s(t)=A_{5}+D_{5}+D_{4}+D_{3}+D_{2}+D_{1}$

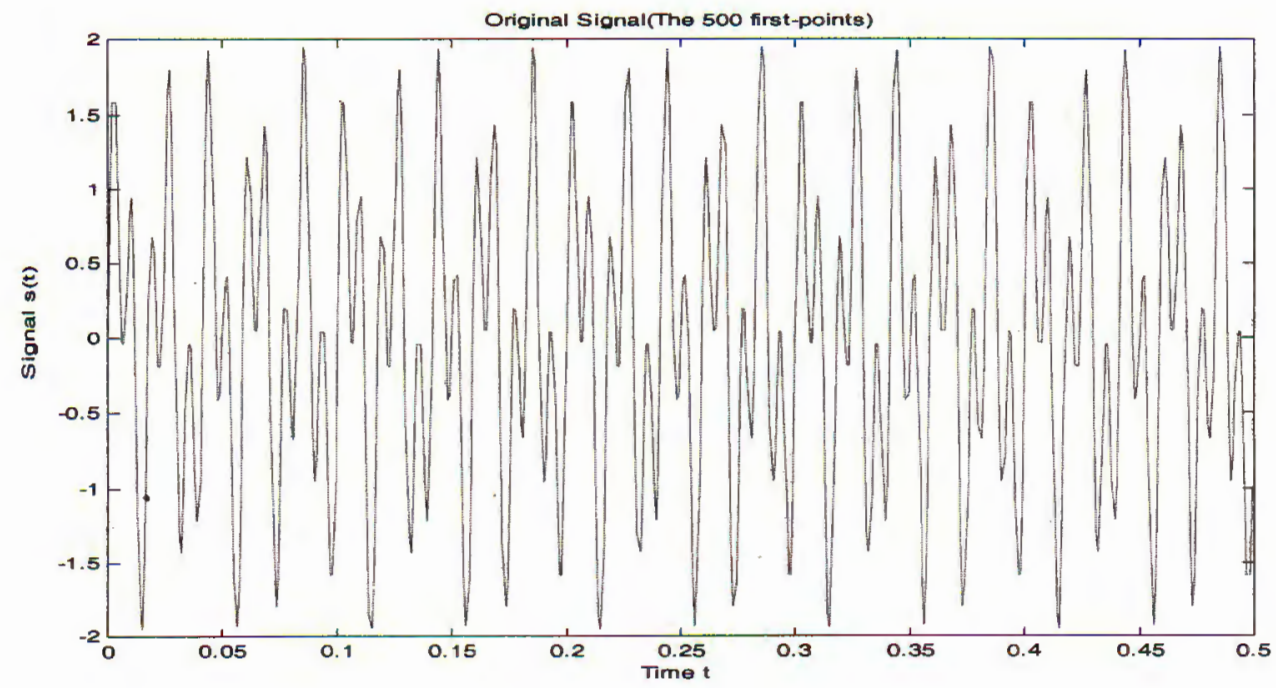

Fig..1. Initial signal $s(t)$

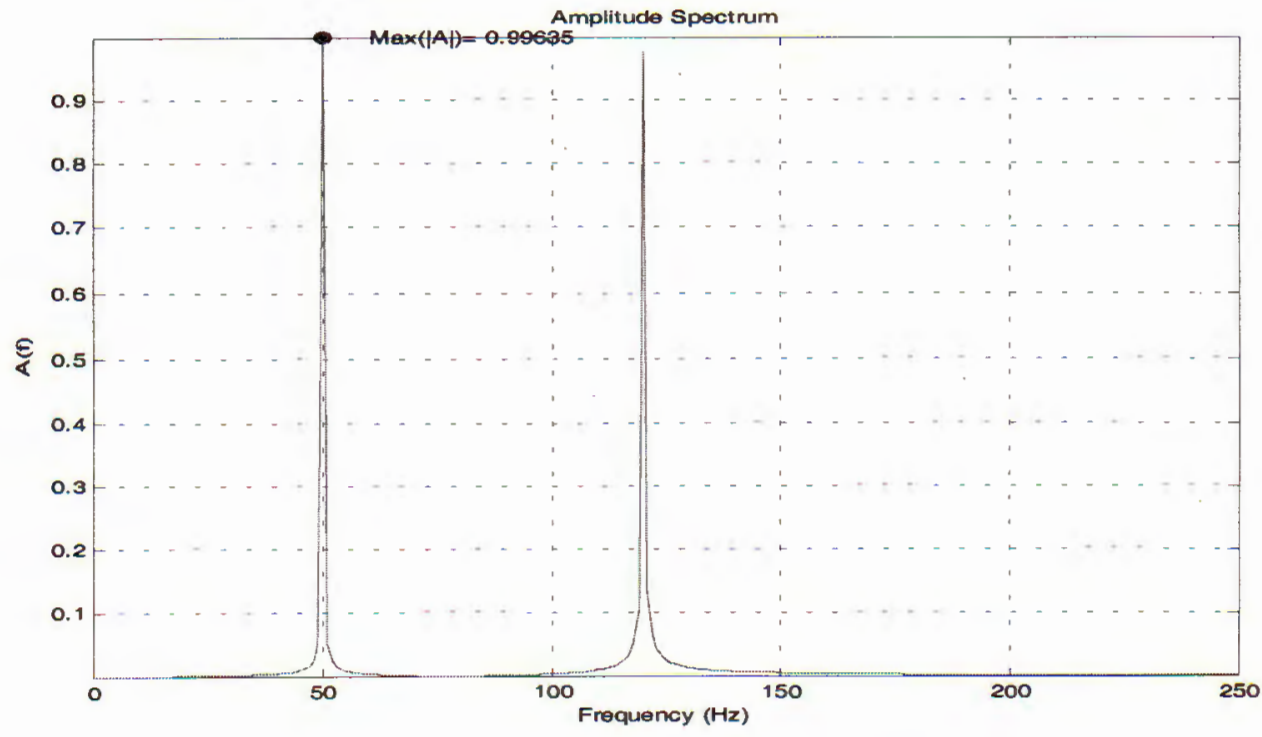

Fig. 2. Amplitude Spectra $|A(f)|$ of signal $s(t)$ 


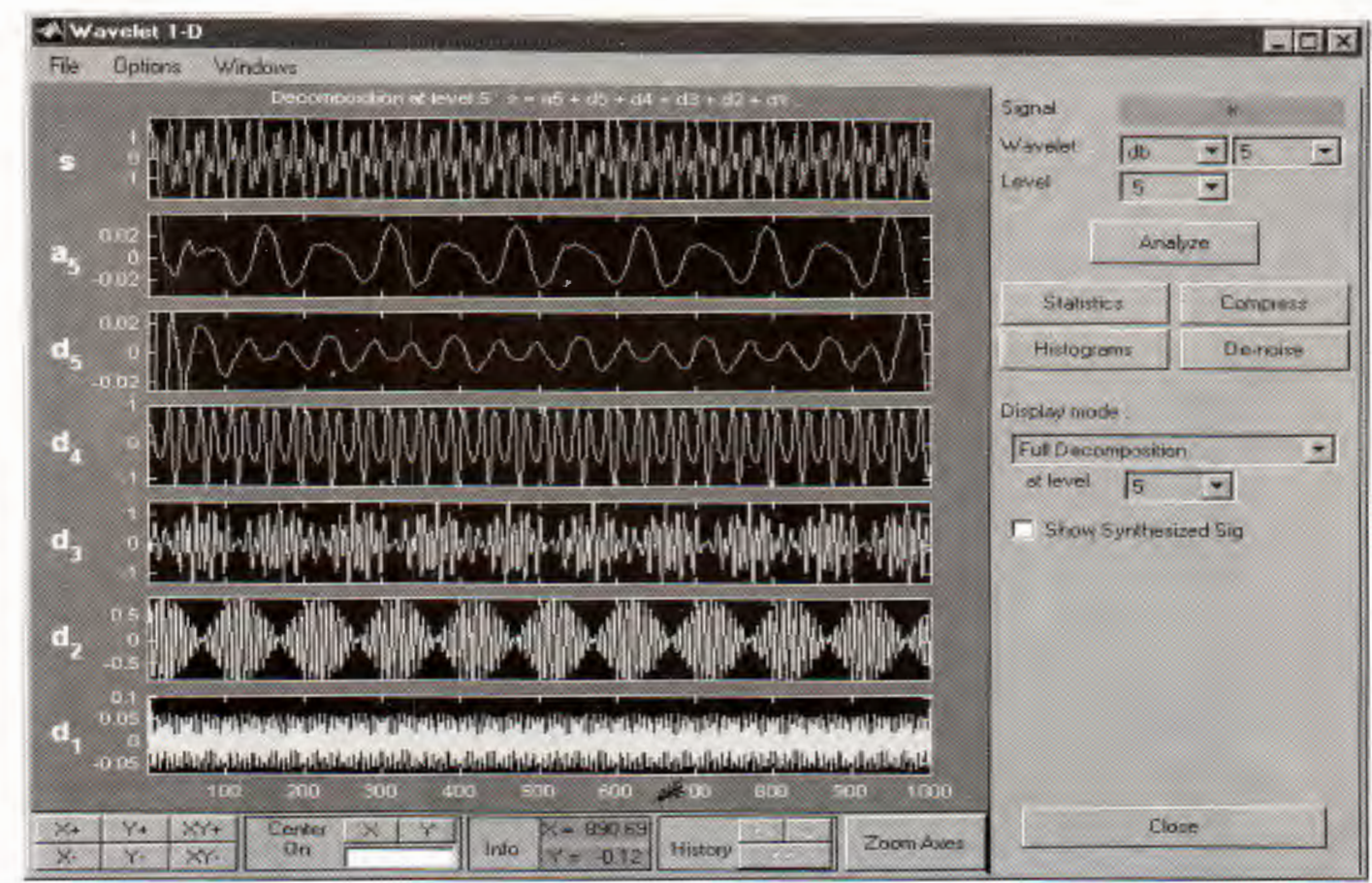

Fig. 3. Graphics for analyzing the signal $s(t)$ using wavelet transform

2. We cannot divide a time interval of signal into parts in which the signal oscillates with the difference frequencies, respectively. However, $A_{5}$ and $D_{5}$ excluded because the vibration amplitudes in $A_{5}, D_{5}$ had been very small comparing with the other vibration amplitudes of the signal. The main vibration properties have to be detected in the components $D_{4}, D_{3}, D_{2}$ and $D_{1}$.

Zooming in the signal part (100 units of time) and note that 1000 units of time on the graphics in figure 3 correspondent to the actual time interval of the signal, we have the following analyses

- Detail $D_{4}$ is a periodic oscillation and it is a signal component oscillating about 5 complete oscillations. Therefore, $D_{4}$ is a periodic oscillation with frequency $f_{1 a}=1000 *(1 / 20)=50 \mathrm{~Hz}=f_{1} . D_{4}$ can be approximated by component $\sin \left(2 \pi f_{1 a} t\right)$.

- Detail $D_{3}$ is a periodic oscillation and it is a signal component oscillating about 9 complete oscillations. Therefore $D_{3}$ is a periodic oscillation with frequency $f_{2 a}=90 \mathrm{~Hz} . D_{3}$ will be excluded because $s(t)$ has not frequency $f=f_{2 a}$.

- Detail $D_{2}$ is a periodic oscillation and it is a signal component oscillating about 12 complete oscillations. Therefore $D_{2}$ is a periodic oscillation with frequency $f_{3 a}=120 \mathrm{~Hz} .=f_{2} . D_{2}$ can be approximated by component $\sin \left(2 \pi f_{3 a} t\right)$. 
- The next analyzing for $D_{1}$ is excluded because the frequencies of given signal was found sufficiently.

So, we have $f_{1 a}=50 \mathrm{~Hz}=f_{1}, f_{2 a}=120 \mathrm{~Hz}=f_{2}$ and then $s(t)$ can be approached by two wavelet components $D_{4}$ and $D_{2}$. Finally, signal $s(t)$ is a periodic oscillation with frequencies $f_{1 a}, f_{2 a}$, which act over time domain of the signal.

The computational results using STFT are presented in figure 4 .

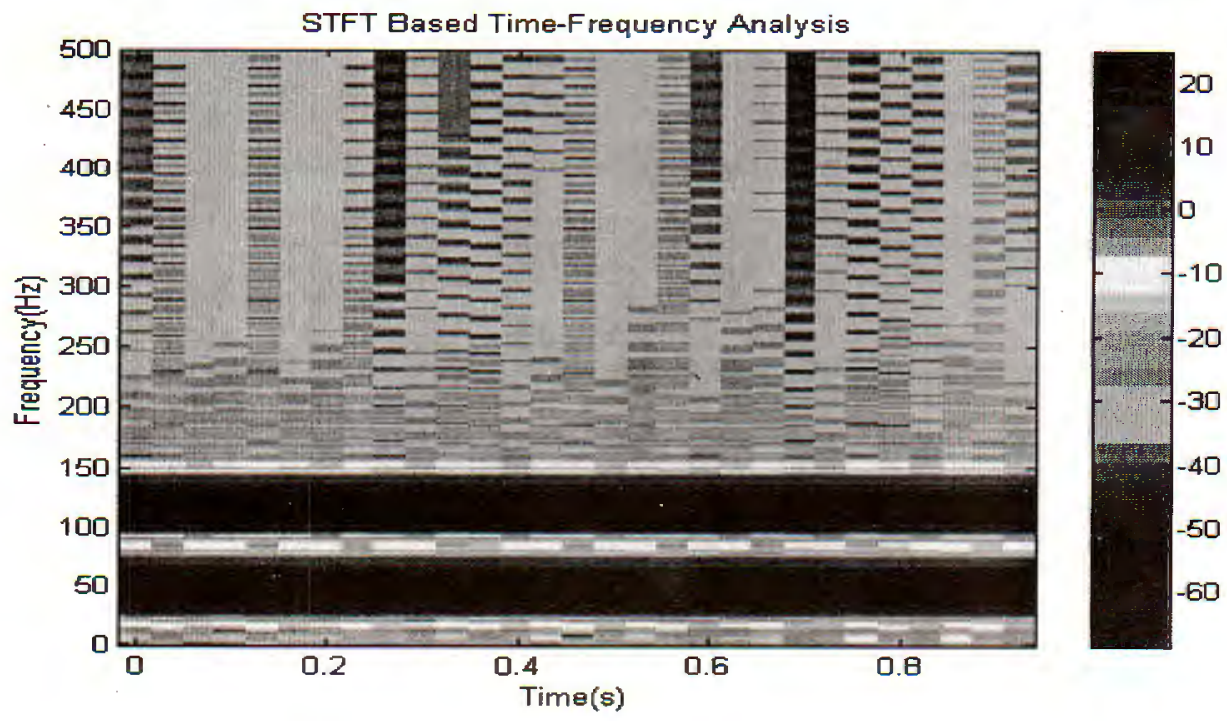

Fig. 4. Time-frequency relation of signal

In figure 4 we obtain two bold red bands. They are the parallel lines with axis of time. Specially, these bold red bands cross exactly the axis of frequency at the frequencies $f_{1 b}=50 \mathrm{~Hz}=f_{1}, f_{2 b}=120 \mathrm{~Hz}=f_{2}$. These red bands are the stretch in all time of a signal. Thus, the signal $s(t)$ acts primarily on the two frequencies $f_{1}$, $f_{2}$ in over time domain of the signal

Example 3.1.2. The signal

$$
s(t)= \begin{cases}\sin (2 \pi 50 t) & \text { with } 0 \leq t \leq 0.4 \\ \sin (2 \pi 120 t) & \text { with } 0.4<t \leq 0.8 \\ \sin (2 \pi 150 t) & \text { with } 0.8<t \leq 1.2\end{cases}
$$

is considered for all $t \in D=[0,1.2]$. The time domain $D$ is divided into three parts. These parts are equal each other. The signal $s(t)$ is the sinusoid harmonic in the each part. It has the correspondent frequencies $f_{1}=50 \mathrm{~Hz}, f_{2}=120 \mathrm{~Hz}$, and $f_{3}=150 \mathrm{~Hz}$.

We shall choose the sampling frequency $f_{C}=1000 \mathrm{~Hz}$ and time $(s) t \in[0,1.2]$.

PC program ARFT322.m give us the results in figures 5, 6, 7 and 8. 
The amplitude spectra analyzed graphic of signal $s(t)$ is given in figure 6 .

In figure 6 we show the amplitude spectra $|A(f)|$ of signal $s(t)$. This spectra has three major values, $A\left(f_{1}\right) \approx 0.33337, A\left(f_{2}\right) \approx 0.33337, A\left(f_{3}\right) \approx 0.33337$. They are correspondent with the frequencies $f_{1}=50 \mathrm{~Hz}, f_{2}=120 \mathrm{~Hz}$ and $f_{3}=150 \mathrm{~Hz}$. So the given signal $s(t)$ has the frequency components $f_{1}=50 \mathrm{~Hz}$ and $f_{2}=120 \mathrm{~Hz}$ and $f_{3}=150 \mathrm{~Hz}$. The wavelet analyzed graphics are presented in figure 7 .

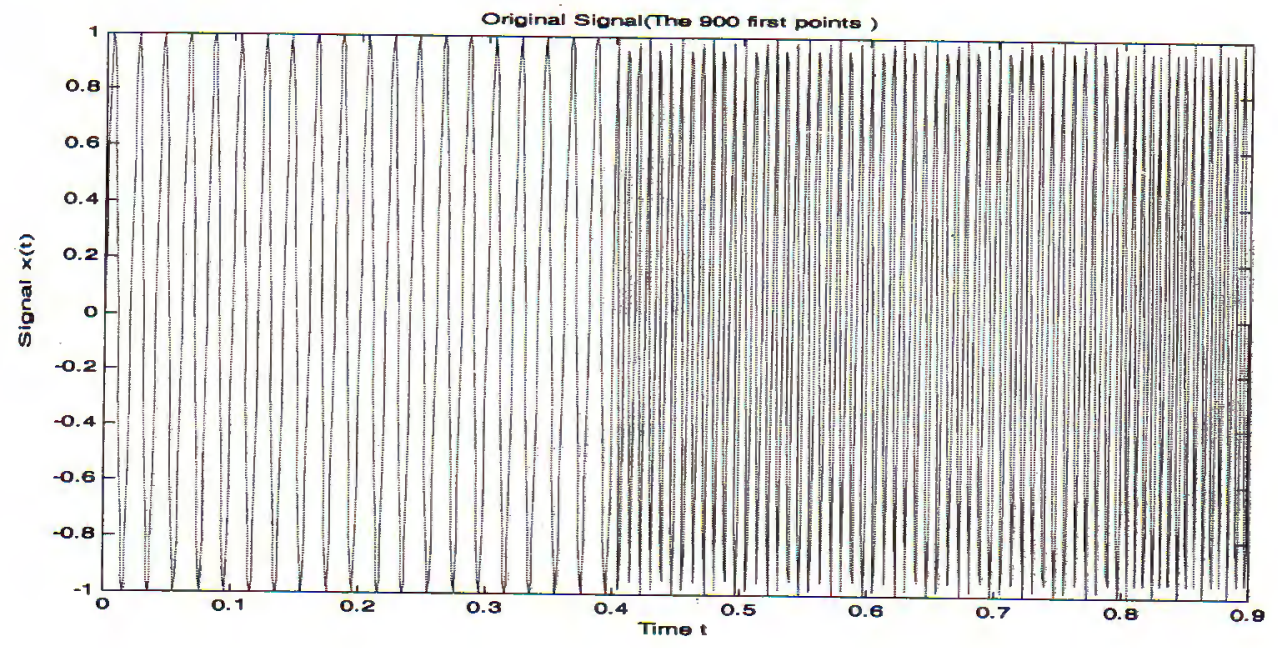

Fig. 5. Initial signal $s(t)$

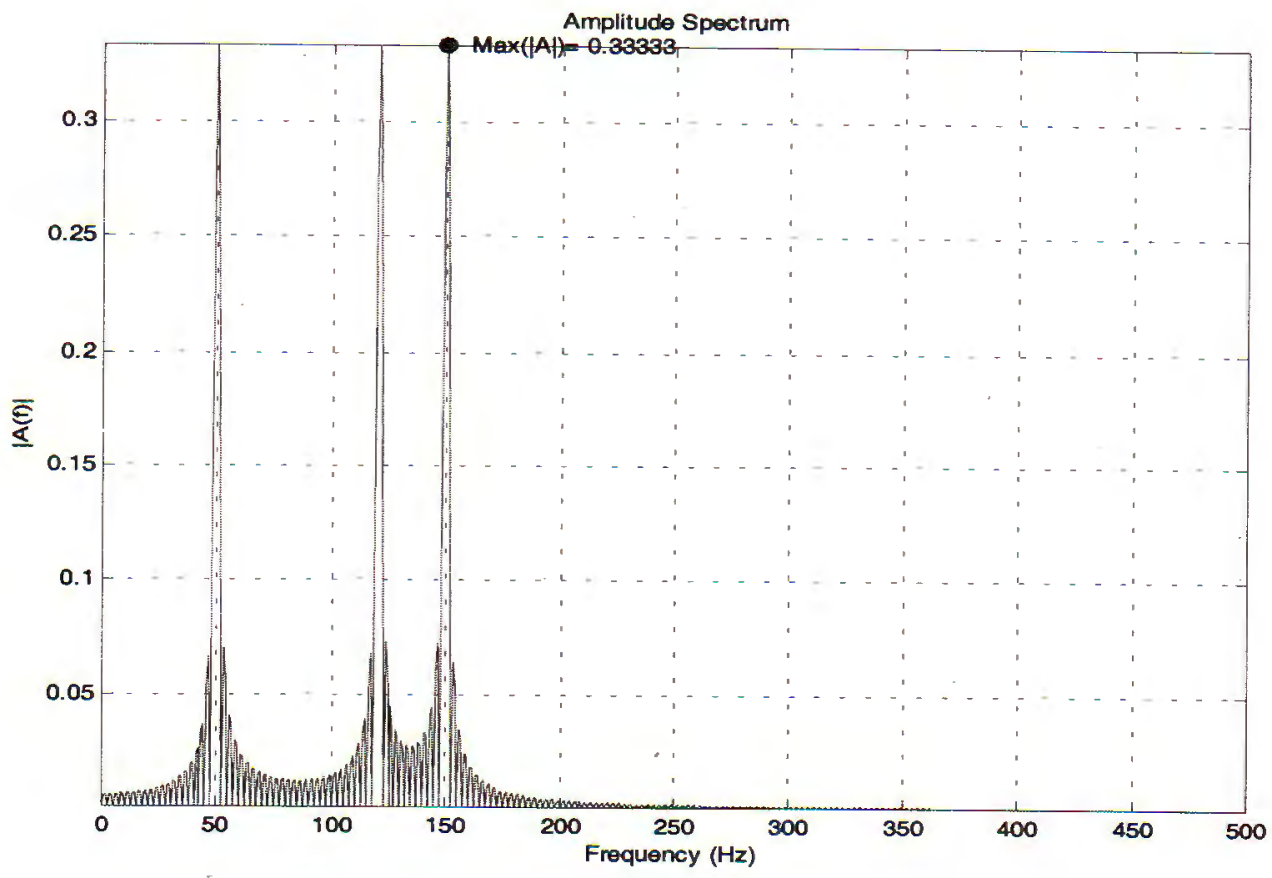

Fig. 6. Amplitude spectra $|A(f)|$ of signal $s(t)$ 


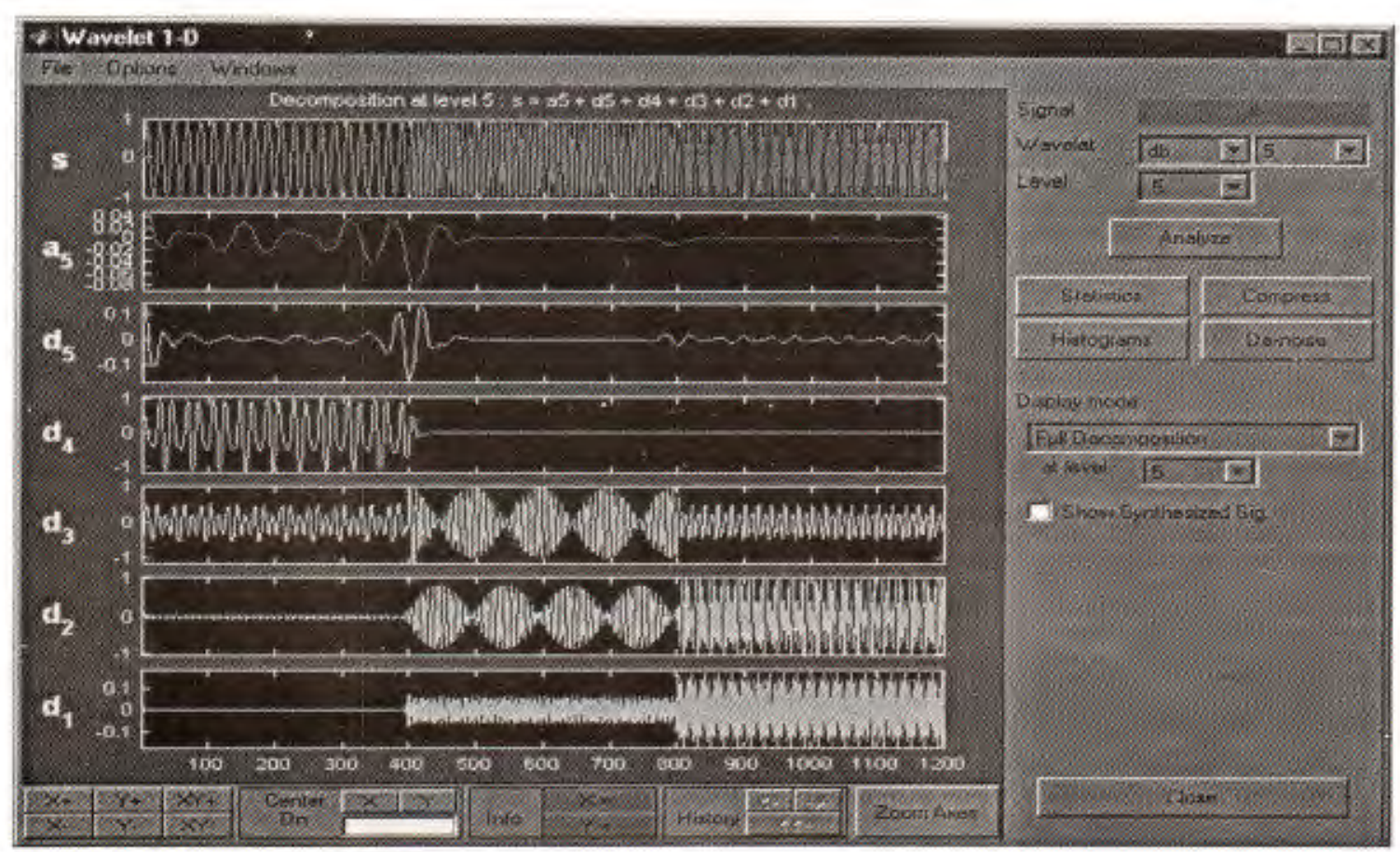

Fig. 7. Graphics for analyzing the signal $s(t)$ using wavelet transform

In figure 7 , we show that:

1. The oscillation of the signal is decomposed into the sum of wavelet components:

$$
s(t)=A_{5}+D_{1}+D_{2}+D_{3}+D_{4}+D_{5} .
$$

2. The forms of oscillation of the signal are very different on three time intervals. Each of intervals contains 400 units of time.

- On the first part of time interval of signal $(1 \leq t \leq 400)$ :

Because the vibration amplitudes in $A_{5}, D_{5}, D_{2}$ and $D_{1}$ had been very small comparing with zero (or they had been very small comparing with the other vibration amplitudes in $D_{4}$ and $D_{3}$ ) then they can be excluded. So, $D_{4}+D_{3}$ is used to replace to the signal $s(t)$. On the first 400 units of time, $D_{4}$ realizes a periodic oscillation with 5 complete oscillations per 100 units of time. Therefore $D_{4}$ oscillates periodically with frequency $f_{1 b}=1000 *(5 / 100)=50 \mathrm{~Hz}$. So, $f_{1 b}=f_{1}$ and $f_{1 b}$ is selected.

Similarly, $D_{3}$ is periodic oscillation with frequency $f_{1 c}=70 \mathrm{~Hz}$, which is not identical with the found frequencies from the amplitude spectrum analysis. Therefore, frequency $f_{1 c}$ is excluded in $D_{3}$.

- On the middle part of time interval of signal $(400<t \leq 800)$ :

Component oscillations $A_{5}, D_{5}, D_{4}$ and $D_{1}$ are excluded because they have small 
amplitudes comparing with the other vibration amplitudes in $D_{3}, D_{2}$. On this time interval, $D_{3}$ oscillates periodically with frequency $f_{2 b}=120 \mathrm{~Hz}=f_{3}$. So that the frequencies in $D_{2}$ become rejects. The second found frequency $f_{2 b}=120 \mathrm{~Hz}$ shows that the given signal $s(t)$ oscillates with this frequency on the middle time interval.

- On the end part of time interval of the $s(t)(800<t \leq 1200)$ :

Component oscillations $A_{5}, D_{5}$ and $D_{4}$ become rejects because they have small amplitudes comparing with the other vibration amplitudes in $D_{3}, D_{2}$ and $D_{1}$. On this time interval, $D_{2}$ oscillates periodically with frequency $f_{3 b}=150 \mathrm{~Hz}=f_{3}$. So that the frequencies in $D_{2}$ and $D_{3}$ become rejects. The thirst found frequency $f_{3 b}=150 \mathrm{~Hz}$ shows that the given signal $s(t)$ oscillates with this frequency on the end time interval. So that $f_{1 b}=50 \mathrm{~Hz}=f_{1}, f_{2 b}=120 \mathrm{~Hz}=f_{2}, f_{3 b}=150 \mathrm{~Hz}=f_{3}$ and the signal $s(t)$ oscillates with three frequencies $f_{1 b}, f_{2 b}$ and $f_{3 b}$ on correspondent time intervals. In other work, the frequencies of the given signal $s(t)$ is a piecewise constant function of time variable on whose time interval of the signal.

The computational results using STFT are presented in figure 8

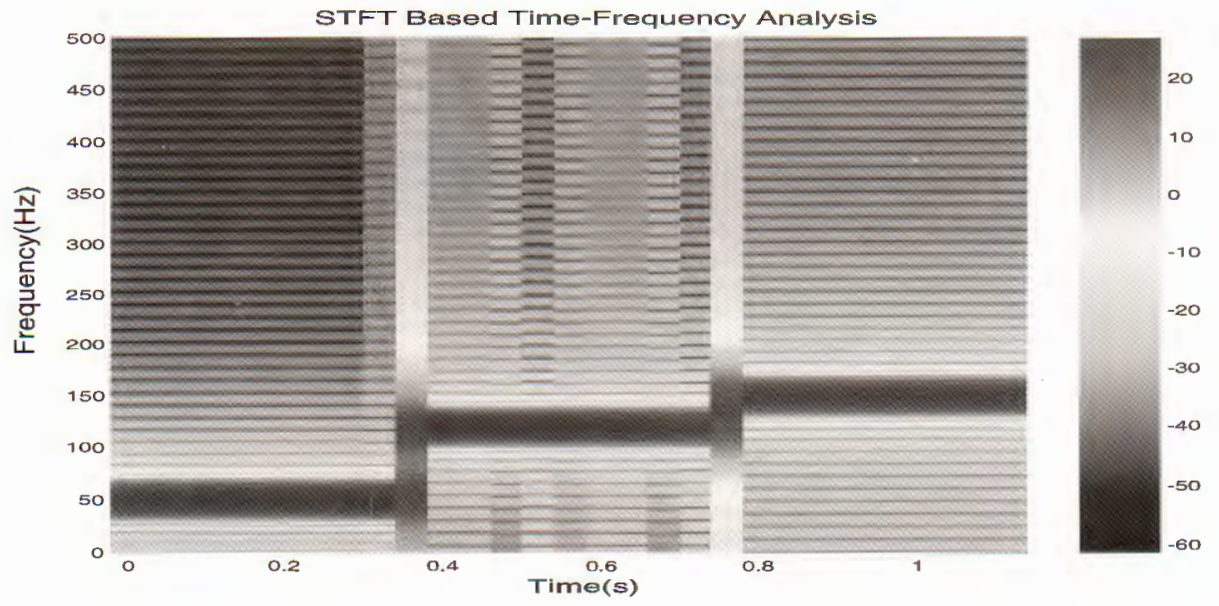

Fig. 8. Time-frequency relation of signal

In figure 8 we obtain three bold red bands. They are the parallel lines with axis of time. Specially, these bold red bands cross exactly the axis of frequency at the frequencies $f_{1}=50 \mathrm{~Hz}, f_{2}=120 \mathrm{~Hz}$ and $f_{3}=150 \mathrm{~Hz}$. These red bands are the stretch in each part of the time domain of the signal. In practice the signal $s(t)$ is the sinusoid harmonic in the each part with the correspondent frequencies: $t \in[0,0.2]$, $f_{1}=50 \mathrm{~Hz} ; t \in[0.2,0.4], f_{2}=120 \mathrm{~Hz} ; t \in[0.4,0.6], f_{3}=150 \mathrm{~Hz}$. Thus, the signal $s(t)$ acts primarily on the three frequencies $f_{1}, f_{2}$ and $f_{3}$ in all of time domain of the signal.

Example 3.1.3. We consider the chirp signal $s(t)=\sin \left(250 \pi t^{2}\right)$.

For this "linear" chirp, the derivative of the phase is linear.

We shall choose the sampling frequency $f_{C}=1000 \mathrm{~Hz}, t \in[0,1.5]$

PC program ARFT323.m gives us the results in figures 9, 10, 11 and 12 . 
Figure 10 shows that, the signal $s(t)$ has only one major values $f_{1} \approx 370 \mathrm{~Hz}$. The wavelet analytical graphics are illustrated in figure 11.

For this signal, use of wavelet analysis to specify time-dependent frequency of given signal is the next research.

The computational results using STFT are presented in figure 12.

In figure 12 the change of the bold red band in time domain of signal is linear. Thus, the frequency of the above mentioned chirp signal $s(t)$ depends linear on time $t$.

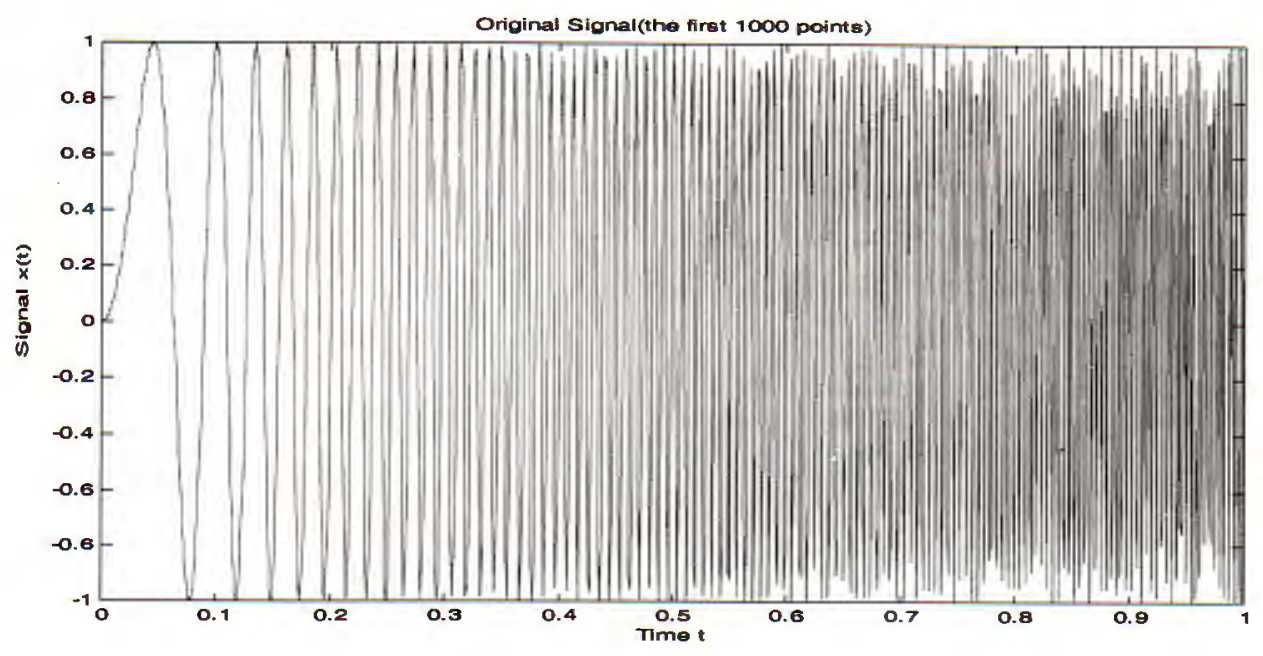

Fig. 9. Chirp signal $s(t)$

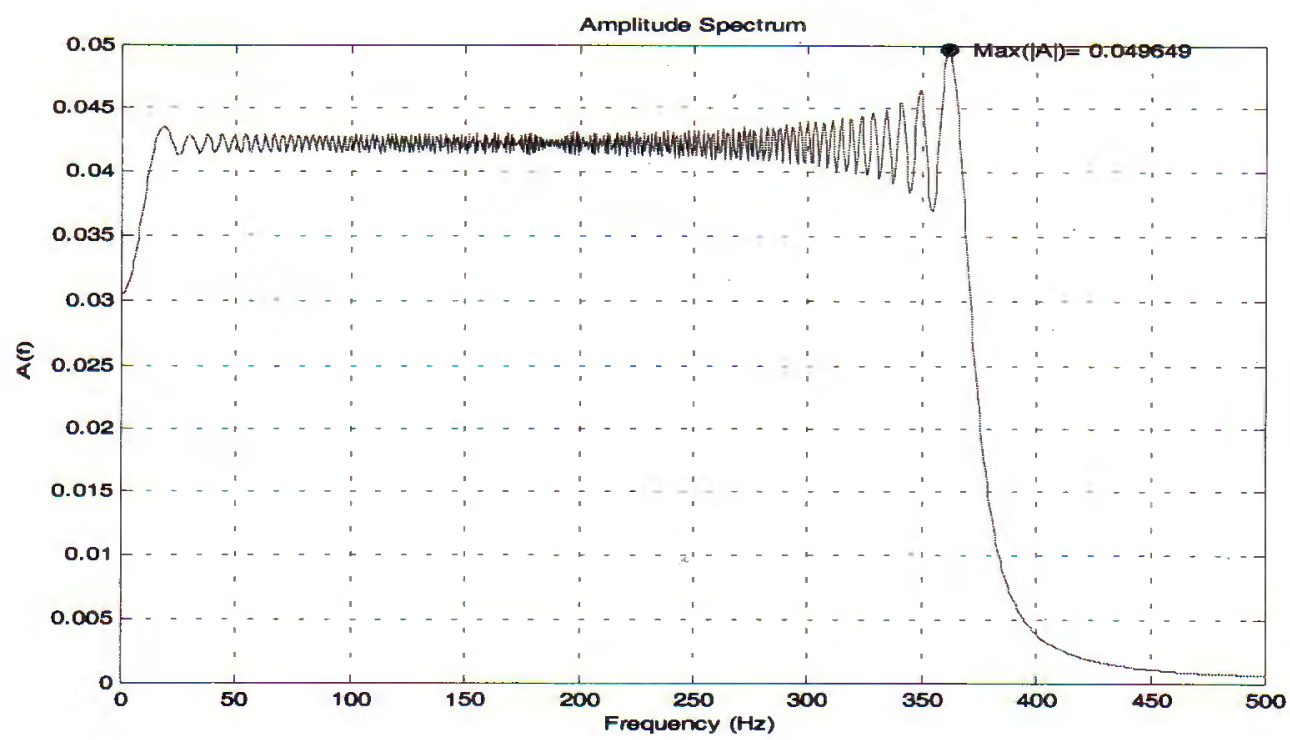

Fig. 10. Amplitude spectra $|A(f)|$ of signal $s(t)$ 


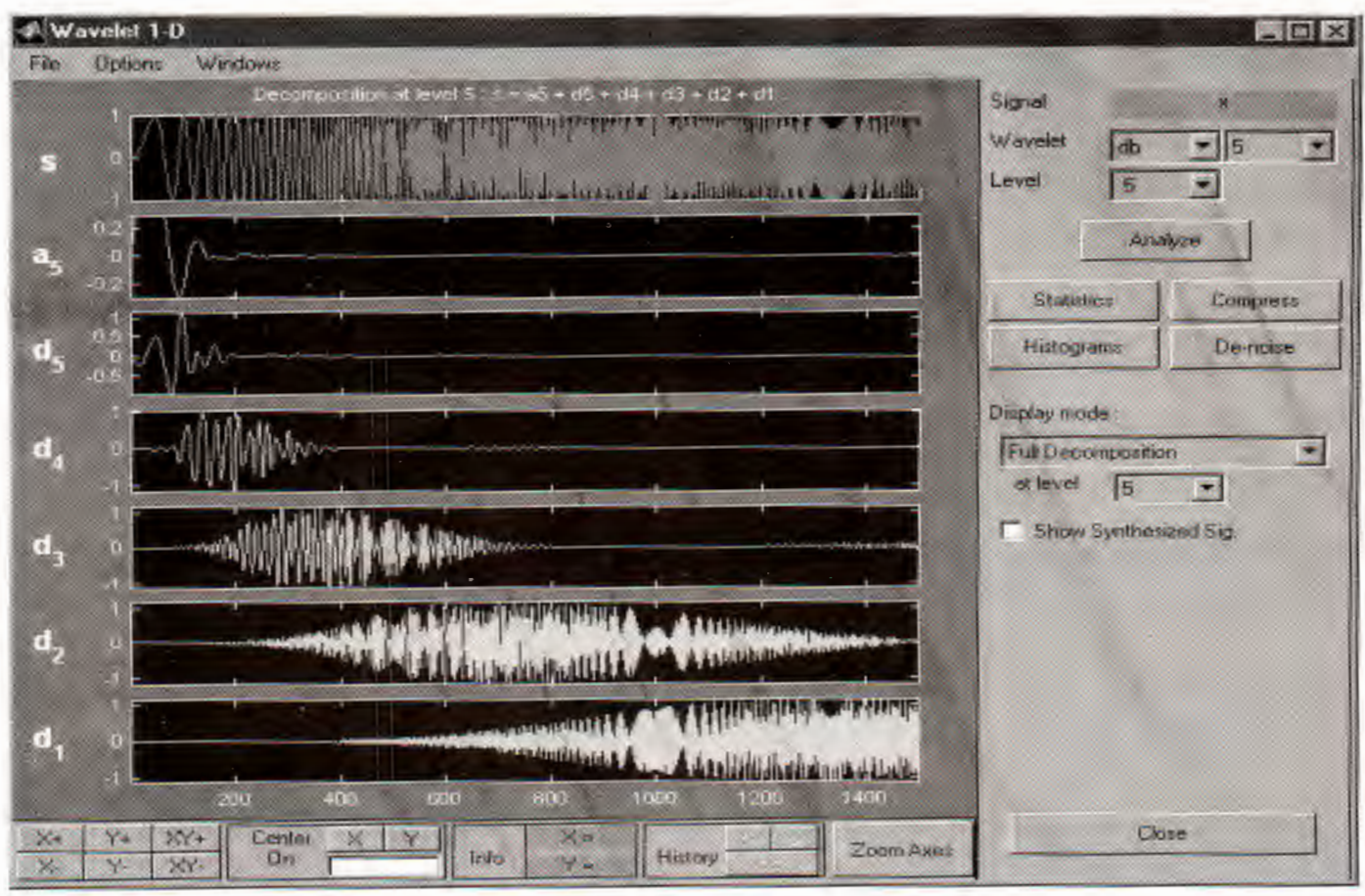

Fig. 11. Graphics for analyzing the signal $s(t)$ using wavelet transform

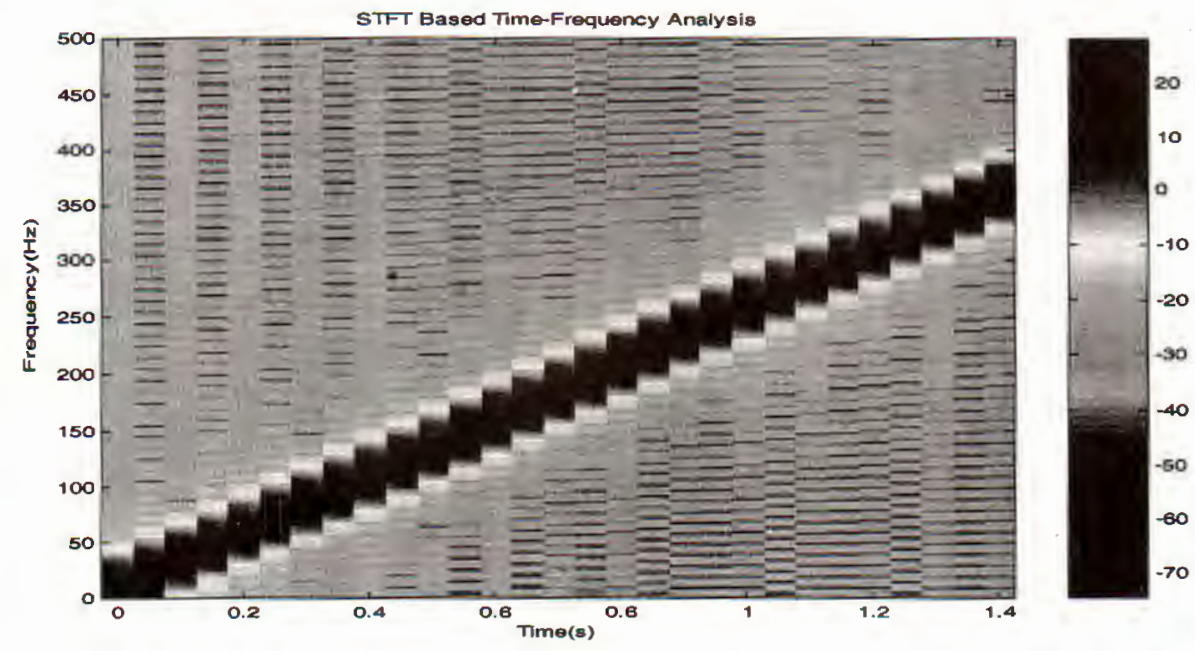

Fig. 12. Time-Frequency relation of the signal $s(t)$

\section{Conclusion}

The resolutions for analyzing the time-frequency relations in vibration signal had been presented. These resolutions base on applications of wavelet analysis. The 
results from spectral analysis is used to support. Short Time Fourier analyses had been used here. The analyses have been explained with the support of computer programs on PC. The results of calculations of examples show that they can be used for analysis well.

This work had completed with the financial support of the Council for Natural Science of Vietnam.

\section{REFERENCES}

1. Louis A. K., Maaß P., Rieder A. Wavelets Theory and Applications. John Wiley and Sons. Inc. New York. 1998.

2. Ingrid Daubechies; Ten Lectures on Wavelets. Society for Industrial and Applied Mathematics. Philadelphia Pennsylvania.1992.

3. Michel Misiti, Yves Misiti. Georges Oppenheim; Jean-Michel Poggi; Wavelet Toolbox For Use with MATLAB. The MathWorks, Inc. 1997.

4. Signal Processing Toolbox For Use with MATLAB. The MathWorks, Inc. 1998.

Received March 29, 2001

\section{MộT VÀI KẾT QUẢ PHÂN TÍCH TẦN SỐ PHỤ THUỘC THOั̀ GIAN TRONG TÍN HIÊU DAO ĐỘNG}

Bài báo này trình bày một vài kết quá phân tích mối quan hệ tần số - thời gian của tín hiệu. Các kết quả nhận được khi nghiên cứu biến đổi wavelet kết hợp với phương pháp phân tích phồ. Các kết quả tương tự khi dùng biến đổi STFT được trình bày ở đây chî với mục đích so sánh. Các chương trình $\mathrm{PC}$ tương ứng được tạo ra nhằm thực hiện giải thuật đồng thời để minh họa các kết quả thông qua khảo sát một vài ví dụ.

* Institute of Mechanics, 264 Doi Can, Hanoi, Vietnam

* E-mail Address: tdtri@im01.ac.vn 